\title{
Protection Motivation Theory and Rabies Protective Behaviors Among School Students in Chonburi Province, Thailand
}

\author{
Mayurin Laorujisawat, Aimutcha Wattanaburanon, Pajaree Abdullakasim, Nipa Maharachpong \\ Faculty of Public Health, Burapha University, Chonburi, Thailand
}

Objectives: The aim of this study was to predict rabies protective behaviors (RPB) based on protection motivation theory (PMT) among fourth-grade students at schools in Chonburi Province, Thailand.

Methods: This cross-sectional study was conducted from December 2020 to February 2021. A multistage sampling technique was used for sample selection. The questionnaire was divided into socio-demographic data and questions related to PMT and RPB. Descriptive statistical analysis was conducted using the EpiData program and inferential statistics, and the results were tested using the partial least squares model with a significance level of less than $5 \%$.

Results: In total, 287 subjects were included, of whom $62.4 \%$ were girls and $40.4 \%$ reported that YouTube was their favorite media platform. Most participants had good perceived vulnerability, response efficacy, and self efficacy levels related to rabies $(43.9,68.6$, and $73.2 \%$, respectively). However, $54.5 \%$ had only fair perceived severity levels related to rabies. Significant positive correlations were found between RPB and the PMT constructs related to rabies $(\beta, 0.298 ; p<0.001)$, and the school variable (S4) was also a predictor of RPB $(\beta,-0.228 ; p<0.001)$. Among the PMT constructs, self efficacy was the strongest predictor of RPB $(\beta, 0.741 ; p<0.001)$.

Conclusions: PMT is a useful framework for predicting RPB. Future RPB or prevention/protection intervention studies based on PMT should focus on improving self efficacy and response efficacy, with a particular focus on teaching students not to intervene with fighting animals. The most influential PMT constructs can be used for designing tools and implementing and evaluating future educational interventions to prevent rabies in children.

Key words: Rabies, Protective behaviors, Protection motivation theory, Students, Thailand

\section{INTRODUCTION}

Rabies is a zoonotic viral disease that affects mammals. Throughout the world, approximately 59000 people are reported to

Received: August 17, 2021 Accepted: October 1, 2021

Corresponding author: Nipa Maharachpong

Faculty of Public Health, Burapha University, 169 Long Hard-Bangsaen Road, Tambon Saensuk, Mueang District, Chonburi 20131, Thailand

E-mail: nipam@go.buu.ac.th

This is an Open Access article distributed under the terms of the Creative Commons Attribution Non-Commercial License (https://creativecommons.org/licenses/bync/4.0// which permits unrestricted non-commercial use, distribution, and reproduction in any medium, provided the original work is properly cited. die from rabies every year [1]. Rabies is present on all continents except Antarctica, and over $95 \%$ of human deaths from rabies occur in Asia and Africa [2]. Once clinical symptoms begin to appear, rabies is almost always fatal [1]. Among zoonotic diseases, the significance of rabies as a public health issue is often neglected [2]. In Thailand, rabies is endemic, and, in most cases (93\%), dogs are the main reservoir [3]. It is estimated that half a million incursions with mammals that could potentially transmit the disease occur annually [4]. The most vulnerable population is children under 15 years of age [4]. From 2011 to 2020, Thailand's eastern region reported the highest incidence of rabies, contributing $36 \%$ of total rabies-related 
deaths in Thailand [3]. In addition, none of the deceased had received any form of treatment [4].

The reason that the most affected group is children under 15 years of age is likely their natural affinity for animals, particularly cats and dogs. Moreover, children generally do not know when they are at risk of rabies. Sometimes children may provoke animals to bite them as a consequence of stone-throwing, beating, chasing, or running at the sight of the animals [5]. In addition, in some cases, children do not pursue any further treatment after the initial treatment of the wound. In contrast, adults are generally more aware of the risk of rabies and tend to seek medical care [6]. A review of the literature [7-10] found that few children were aware that rabies was a potentially fatal disease and understood that they were vulnerable to the disease. Therefore, awareness and education are key. In addition, the cognitive process plays a key role in decision-making and influencing behavioral changes [11], the factors of which correspond to protection motivation theory (PMT).

PMT has been widely adopted as a framework for predicting and influencing health-related behaviors [12]. PMT is based on 2 cognitive processes. The first is threat appraisal (TA), which consists of 2 cognitive aspects - perceived severity (PS) and perceived vulnerability (PV) - and can influence changes in attitudes and behaviors. The second cognitive process is coping appraisal (CA), which also consists of 2 cognitive aspectsresponse efficacy (RE) and self-efficacy (SE) — and can influence individuals to successfully avoid danger, which is an important factor for making positive behavioral changes. These factors are influenced by fear-inducing media rather than more standard methods of persuasion such as external stimuli, environmental organization, persuasion, observational learning, personality, and experience [13].

Researchers have used PMT to investigate and understand a wide range of health-related behaviors and preventive/protective behaviors [14-16]. In addition, PMT has been used in etiological studies to investigate various risk behaviors in children, including tobacco or drug use [17-19], sexual initiation perceptions [20-22], and cybersecurity behavior [23]. Historically, few studies have been conducted examining PMT in the context of rabies prevention, which is one of this study's main objectives. Moreover, the age of 9 years to 10 years old is a good starting point for providing education related to selfcare behaviors [24]. At this age, children are able to develop a thorough understanding of these issues due to their developmental stage. Through knowledge and reasoning, children can develop greater healthcare awareness [25].

Partial least squares structural equation modeling (PLS-SEM) is one of the most widely used multivariate data analysis methods in the business and social science fields, including in education, psychology, health, and nursing. It is primarily used to examine models with latent variables [26]. Standard PLS-SEM analyses provide information on the relative importance of constructs for explaining other constructs in a structural model. Information on the importance of constructs is relevant for drawing conclusions. Importance-performance map analysis (IPMA) extends the results of PLS-SEM by also examining the performance of each construct. As a result, conclusions can be drawn across 2 dimensions (both importance and performance), which is particularly important for understanding which managerial actions to prioritize [27], and the analysis can be expanded to the indicator level to facilitate identification of the most important aspects of specific actions [28]. Due to its simplicity and practicality, importance-performance analysis has been popular among researchers and professionals involved in customer satisfaction and quality management for promoting efficient resource utilization in various fields, including health [28-30].

This study was conducted to predict the rabies protective behaviors (RPB) of fourth-grade students by applying PMT and using PLS-SEM and IPMA to close an existing gap in the research, with the ultimate aim of improving children's RPB. Upon the successful completion of PMT analysis, the data can be used to devise targeted rabies prevention programs for elementary school children.

\section{METHODS}

\section{Participants and Sampling}

This cross-sectional study analyzing RPB among fourth-grade students was conducted from December 2020 to February 2021. It was conducted with the permission of the children and their guardians. The number of participants in this study was calculated with $G^{*}$ Power 3.1 [31] by selecting the $F$-test with linear multiple regression, a fixed model, and the $R^{2}$ deviation from zero option. The sample size was calculated using a 95\% confidence interval $(\mathrm{Cl})$ and a $5 \%$ error rate. As a result, the sample size was determined to be 270 participants, and we planned for a possible $5 \%$ non-response rate. In total, 287 subjects were included. A multistage sampling technique was used for selecting the sample units. The 11 districts in Chon- 
buri Province were divided into 2 strata, with the first consisting of 4 districts with a recent history of dogs infected with rabies being found in 2020 and the second consisting of 7 districts with no such history. Three sub-districts were randomly selected from each stratum, for a total of 6 sub-districts. In the second step, 1 school was randomly selected from each subdistrict, except for in Nong Samsak, where there were only very small schools. Thus, 2 schools were selected from Nong Samsak, for a total of 7 schools. In the third step, fourth-grade students were randomly sampled using the lottery method. After the data were organized and verified, a total of 287 fourthgrade students were selected from 7 primary schools across 6 sub-districts of Chonburi Province.

\section{Research Instruments}

A rabies questionnaire was developed based on academically reviewed information on rabies, PMT, and prevention or protective behaviors among children. The research instrument was a closed-ended questionnaire divided into 3 sections on (1) socio-demographic data, (2) PMT covering 4 areas (PS, PV, $\mathrm{RE}$, and SE), and (3) RPB.

The final version of the questionnaire had an average content validity of more than 0.6 , which was calculated using itemobjective congruence and indicated adequate content validity. The reliability was measured using Cronbach's alpha coefficient, which was 0.806 for PMT and 0.773 for RPB in this study.

\section{Statistical Analysis}

The data collected from the surveys were organized and verified for completeness and entered into EpiData Analysis version 2.1 (https://www.epidata.dk/). Descriptive statistics were calculated for (1) socio-demographic data, (2) the variables related to PMT, and (3) RPB. The PMT constructs were categorized according to Bloom's cut-off points ( $<60$ indicating poor, $60-80$ indicating fair, and $>80 \%$ indicating good) [32]. Inferential statistics were tested using PLS-SEM with a significance level of less than $5 \%$.

\section{Ethics Statement}

This study was conducted under the highest ethical standards as outlined by the Burapha University-Institutional Review Board for Protection of Human Subject in Research (BUUIRB), Thailand (\#079/2563). The participants' identifying information remained confidential throughout.

\section{RESULTS}

\section{Socio-demographic Characteristics of the Students}

Table 1 shows that a total of 287 fourth-grade students participated in the study from 7 primary schools across 6 sub-districts of Chonburi Province. The majority of the participants were girls (62.4\%). The proportion of subjects from schools 1 through 7 were $17.8 \%, 9.4 \%, 5.2 \%, 18.5 \%, 19.9 \%, 15.3 \%$, and $13.9 \%$, respectively. Among the participants, $50.9 \%$ lived in areas where infected dogs were found, and $47.3 \%$ of their fathers and $38.3 \%$ of their mothers reported being self-employed. The most popular media platforms were YouTube (40.4\%) and TikTok (36.9\%), and $26.5 \%$ of the participants reported that they spent between 1 hour and 2 hours per day consuming content on their preferred media platform. A total of $60.3 \%$ of the participants had a pet, of which the majority were dogs $(42.9 \%)$, with an average number of $1.26 \pm 2.30$ dogs. More than half (53.3\%) of the participants had no history of being bitten, scratched, or licked at a wound site by a mammal. Of the participants who reported being injured by a mammal, $34.5 \%$ were scratched, $21.3 \%$ were bitten, and $3.8 \%$ were licked at the wound.

\section{Protection Motivation Theory Constructs and Rabies Protective Behaviors}

The PMT construct scores were classified using Bloom's cutoff points. The data showed that $61.3 \%$ of participants had a good level of PMT constructs. When considering each of the aspects of PMT, it was found that the participants had good $P V, R E$, and SE related to rabies $(43.9,68.6$, and $73.2 \%$, respectively). Only PS had a fair level, at $54.5 \%$. As shown in Table 2, most of the respondents were unsure that rabies is a fatal disease $(60.6 \%)$, causes brain damage (65.9\%), and typically kills infected animals within 10 days (65.9\%). A total of $52.3 \%$ of the participants had a good level of RPB; however, $51.9 \%$ answered that they would not encourage local individuals to annually vaccinate their pets (PB1).

Figure 1 and Table 3 present the results of PLS-SEM. Significant positive correlations were found between RPB and the PMT constructs related to rabies $(\beta, 0.298$; standard error [SE], $0.053 ; p<0.001)$, and the school, particularly school No. 4 (S4), was a predictor of RPB $(\beta,-0.228 ; \mathrm{SE}, 0.058 ; p<0.001)$. Students in $\mathrm{S} 4$ had lower RPB scores than students in other schools.

The present study showed that the students perceived that 
Table 1. Demographic information

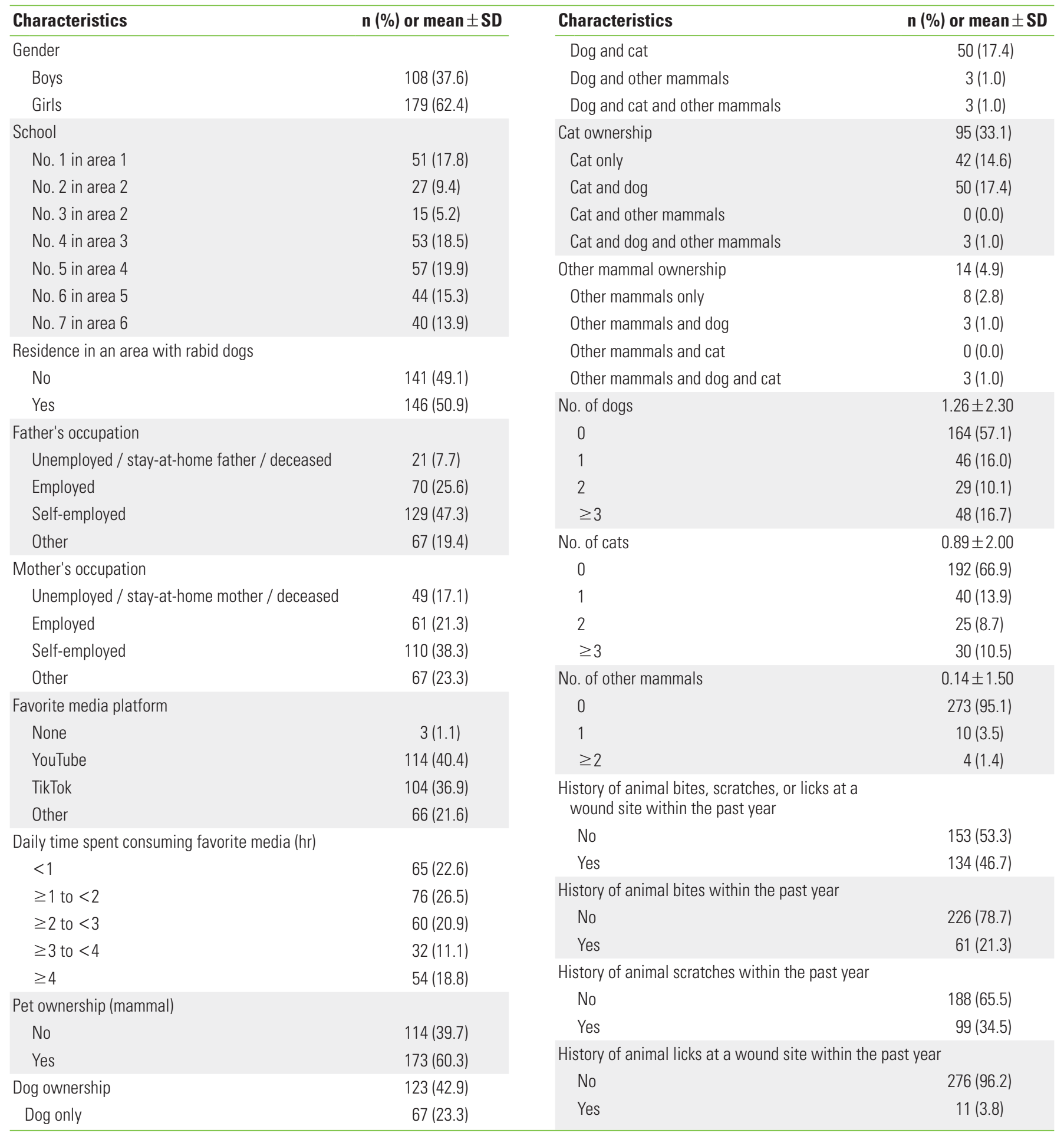

wash the wound with running water and soap several times for at least 15 minutes could mitigate the risk of contracting rabies (PV3), with the PV3 indicator significantly correlating with PMT constructs $(\beta, 0.098 ; \mathrm{SE}, 0.024 ; p<0.001)$. In addition, the media $(\beta, 0.160 ; \mathrm{SE}, 0.061 ; p=0.009)$ and gender indicators $(\beta,-0.160 ; \mathrm{SE}, 0.066 ; p=0.015)$ were found to be significantly associated with $P V$, meaning that a preference for YouTube affected the PV score more than other media platforms and that 
Table 2. Questions related to protection motivation theory and rabies and rabies preventive behaviors

\begin{tabular}{|c|c|c|c|}
\hline Variables & Agree & Unsure & Disagree \\
\hline \multicolumn{4}{|l|}{ Perceived severity (PS) } \\
\hline PS1: If you have a rabies infection, you will die & $65(22.6)$ & $174(60.6)$ & $48(16.7)$ \\
\hline PS2: The rabies virus primarily damages the brain & $65(22.6)$ & $189(65.9)$ & $33(11.5)$ \\
\hline PS3: Dogs and cats who are infected with rabies will perish within $10 \mathrm{~d}$ & $46(16.0)$ & $189(65.9)$ & $52(18.1)$ \\
\hline PS4: If you get bitten and infected by a rabid dog and are not medically treated, you will die & $154(53.7)$ & $104(36.2)$ & $29(10.1)$ \\
\hline \multicolumn{4}{|l|}{ Perceived vulnerability (PV) } \\
\hline PV1: You can get rabies if you play with an unknown dog or cat & $95(33.1)$ & $139(48.4)$ & $53(18.5)$ \\
\hline $\begin{array}{l}\text { PV2: If you get scratched by a cat and immediately tell your parents or teachers, you will have a greater chance of } \\
\text { avoiding rabies infection }\end{array}$ & $160(55.7)$ & $65(22.6)$ & $62(21.6)$ \\
\hline $\begin{array}{l}\text { PV3: If after being bitten you wash the wound with clean water and soap several times for at least } 15 \text { min, you will } \\
\text { have a greater chance of not contracting a rabies infection }\end{array}$ & 195 (67.9) & $61(21.3)$ & $31(10.8)$ \\
\hline \multicolumn{4}{|l|}{ Response efficacy (RE) } \\
\hline $\begin{array}{l}\text { RE1: I think that telling a parent or teacher immediately after being scratched by a cat will help to prevent a rabies } \\
\text { infection }\end{array}$ & $162(56.4)$ & $79(27.5)$ & $46(16.0)$ \\
\hline $\begin{array}{l}\text { RE2: I think that informing parents or people about the annual rabies animal vaccination will help to prevent rabies } \\
\text { infection }\end{array}$ & $193(67.2)$ & $75(26.1)$ & $19(6.6)$ \\
\hline RE3: I think avoiding ownerless cats and dogs is a good method to prevent rabies infection & $194(67.6)$ & $53(18.5)$ & $40(13.9)$ \\
\hline RE4: I think not intervening with animals when they are fighting will help to prevent rabies infection & $200(69.7)$ & $53(18.5)$ & $34(11.8)$ \\
\hline \multicolumn{4}{|l|}{ Self-efficacy (SE) } \\
\hline SE1: I am confident that if I do not interfere with unknown animals, I will reduce my risk of contracting rabies & $208(72.5)$ & $59(20.6)$ & $20(7.0)$ \\
\hline $\begin{array}{l}\text { SE2: I am confident that I can reduce my risk of contracting rabies by informing local individuals to vaccinate their } \\
\text { pets }\end{array}$ & $204(71.1)$ & $68(23.7)$ & $15(5.2)$ \\
\hline $\begin{array}{l}\text { SE3: I am confident that if I immediately inform my teachers/parents after being bitten, scratched, or licked by a } \\
\text { mammal, I can reduce my risk of contracting rabies }\end{array}$ & $237(82.6)$ & $33(11.5)$ & $17(5.90)$ \\
\hline SE4: I am confident that if I can identify an animal that scratched me, I can reduce my risk of contracting rabies & $70(24.4)$ & $149(51.9)$ & $68(23.7)$ \\
\hline $\begin{array}{l}\text { SE5: I am confident that if I wash a wound with soap and water for } 15 \mathrm{~min} \text { (WASH15) after being bitten, scratched, } \\
\text { or licked by an unknown animal, I can reduce my risk of contracting rabies }\end{array}$ & $171(59.6)$ & $94(32.7)$ & $22(7.7)$ \\
\hline SE6: I am confident that if I do not intervene with fighting dogs, I can reduce my risk of contracting rabies & $248(86.4)$ & $21(7.3)$ & $18(6.3)$ \\
\hline \multicolumn{4}{|l|}{ Rabies protective behaviors (PB) } \\
\hline PB1: I will inform local individuals to annually vaccinate their pets & $33(11.5)$ & 105 (36.6) & 149 (51.9) \\
\hline $\begin{array}{l}\text { PB2: I will immediately inform teachers/parents if I am bitten or scratched by a mammal even if the wound does not } \\
\text { bleed }\end{array}$ & 114 (39.7) & $115(40.1)$ & $58(20.2)$ \\
\hline $\begin{array}{l}\text { PB3: I will immediately inform teachers/parents if I witness a friend getting bitten, scratched, or licked at a wound } \\
\text { site by a mammal }\end{array}$ & 91 (31.7) & $130(45.3)$ & $66(23.0)$ \\
\hline $\begin{array}{l}\text { PB4: I will wash the wound with soap and water ( } 15 \mathrm{~min} \text { ) if I am bitten, scratched, or licked at a wound site by a } \\
\text { mammal }\end{array}$ & 87 (30.3) & $139(48.4)$ & 61 (21.3) \\
\hline
\end{tabular}

Values are presented as number (\%).

scores for PV were higher among girls than boys.

There were 2 statistically significant indicators of RE: RE2 ('I think that informing parents or people about the annual rabies animal vaccination will help to prevent rabies infection') and RE4 ('I think not intervening with animals when they are fighting will help to prevent rabies infection'), that the students believed could prevent rabies. These showed significant relationships with the other PMT constructs $(\beta, 0.477 ; \mathrm{SE}, 0.019 ; p<0.001)$. Three SE indicators had a statistically significant relationship to PMT constructs $(\beta, 0.741 ; S E, 0.023 ; p<0.001)$, indicating that students believed they were able to perform those behaviors to prevent rabies. They were SE2 ('I am confident that I can reduce my risk of contracting rabies by informing local individuals to vaccinate their pets'), SE3 ('I am confident that if I immediately inform my teachers/parents after being bitten, scratched, or licked by a mammal, I can reduce my risk of contracting rabies'), and SE6 ('I am confident that if I do not intervene with fighting dogs, I can reduce my risk of contracting rabies'). Non-significant relationships were found between PS and the other PMT constructs. 


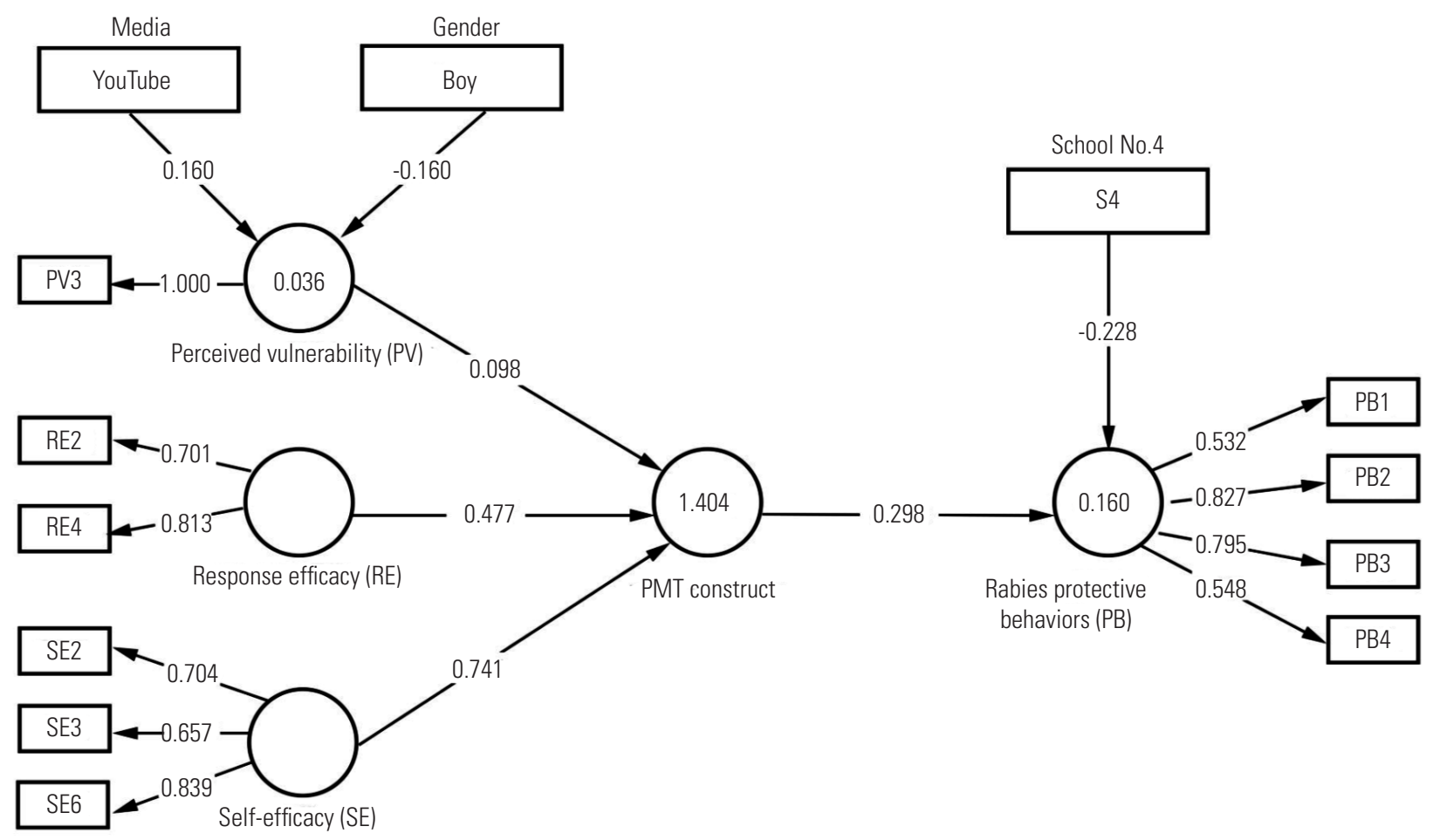

Figure 1. Path diagram of the structural equation model. PMT, protection motivation theory.

Table 3. Detailed measurement and regression results of the structural equation modeling shown in Figure 1

\begin{tabular}{lccc}
\hline Variables & Coefficient & SE & p-value \\
\hline Perceived vulnerability & & & \\
$\quad$ Media & 0.160 & 0.061 & 0.009 \\
$\quad-0.160$ & 0.066 & 0.015 \\
$\quad$ Gender & & & \\
$\quad$ Perceived vulnerability & 0.098 & 0.024 & 0.000 \\
$\quad$ Response efficacy & 0.477 & 0.019 & 0.000 \\
Self efficacy & 0.741 & 0.023 & 0.000 \\
Rabies protective behaviors & & & \\
$\quad$ PMT constructs & 0.298 & 0.053 & 0.000 \\
School No. 4 & -0.228 & 0.058 & 0.000
\end{tabular}

SE, standard error; PMT, protection motivation theory.

\section{Importance Performance Map Analysis}

IPMA analysis was conducted for the variables that affected RPB in the initial analysis. IPMA uses a 2-dimensional grid to plot the mean ratings for performance and importance. The $x$ axis is used to map a variable's importance, measured by the total effect (0-1), and the $y$-axis is used to map a variable's performance, based on an adjusted raw score ranging from 0-100 that corresponds to the percentage value. The resulting 4-quadrant matrix, classified as quadrants $A$ through $D$ (Figure 2), can be used both to identify opportunities for improvement and to guide strategic planning schemes [33]. Three indicators (RE2: 'I think that informing parents or people about the annual rabies animal vaccination will help to prevent rabies infection, SE2:'I am confident that I can reduce my risk of contracting rabies by informing local individuals to vaccinate their pets,' and SE6: I am confident that if I do not intervene with fighting dogs, I can reduce my risk of contracting rabies') were in quadrant $A$, meaning that RE2, SE2, and SE6 had above-average importance and above-average performance.

There were 2 indicators in quadrant B (PV3: 'If after being bitten you wash the wound with clean water and soap several times for at least 15 minutes, you will have a greater chance of not contracting a rabies infection,' and SE3: 'I am confident that if I immediately inform my teachers/parents after being bitten, scratched, or licked by a mammal, I can reduce my risk of contracting rabies'), meaning that the PV3 and SE3 indicators had below-average importance but above-average performance. The gender and media indicators were in quadrant $\mathrm{C}$, meaning that they had below-average importance and performance. Lastly, RE4 ('I think not intervening with animals when they are fighting will help to prevent rabies infection') and S4 were in quadrant $D$, which had high importance but below-average performance. 


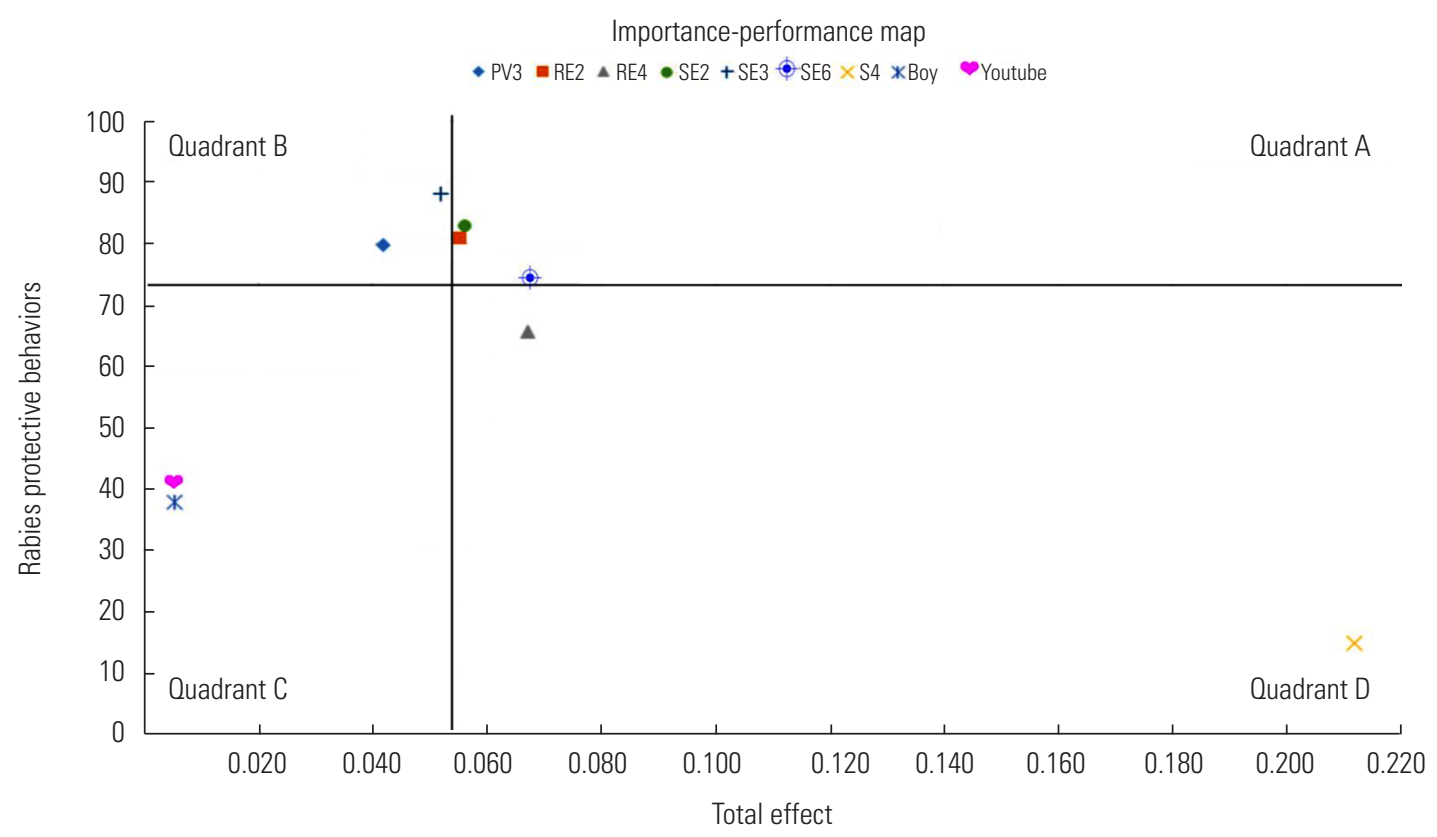

Figure 2. Importance performance map analysis. PV, perceived vulnerability; RE, response efficacy; SE, self-efficacy.

\section{DISCUSSION}

This study used PLS-SEM to predict structural relationships in our model based on PMT and to determine the key factors related to RPB among primary school students. PMT consists of a combination of 4 constructs (PS, PV, RE, and SE). The students were found to have good levels of PV, RE, and SE (43.9, 68.6 , and $73.2 \%$, respectively). The students' overall scores were only fair for PS, at $54.5 \%$. This finding is consistent with the results of a study by Kumsri [34] but inconsistent with 2 other previous studies $[14,35]$. The reason why most fourthgrade students achieved only a moderate level for PS may have been that PS, more than the other constructs, is age-dependent [15], and children and adults are at different stages of cognitive development [23].

According to the results of the path analysis, the PMT constructs $(\beta, 0.298 ; \mathrm{SE}, 0.053 ; p<0.001)$ and the S4 school indicator $(\beta,-0.228 ; \mathrm{SE}, 0.058 ; p<0.001)$ had significant relationships with RPB. The students in S4 had the lowest rate of RPB of the participants. Accordingly, S4 was located in an area with fewer reported rabies outbreaks than in the other areas where schools were located, and teachers and parents may not have been focused on rabies prevention [36].

The study findings also showed that, of the PMT constructs, SE was the strongest predictor $(\beta, 0.741 ; \mathrm{SE}, 0.023 ; p<0.001)$, which is consistent with the findings of previous studies [15,
$18,19,23]$. This positive correlation indicates that individuals with a high SE score can consistently act against rabies, reduce their own risk of rabies, protect their health, and prevent the negative consequences of poor RPB. Therefore, when designing educational interventions, it is essential to emphasize SE to mitigate the threat of rabies [18]. PS, however, was not a significant predictor of PMT constructs and RPB in this study, which is a similar finding to those of some previous studies $[14,17,20,22,37]$ and an inconsistent finding with others $[11,15$, 16,34]. The PS scores of the participants in this study were not high. The mean value was $8.59 \pm 1.57$, and most students were unsure as to the severity of rabies. Nevertheless, PS can be decisive as it relates to PMT constructs since, if students are aware of the consequences and harms of rabies, protection motivation will in turn increase [18]. Therefore, when designing educational programs, it is essential to pay special attention to PS by incorporating anecdotes from children who experienced dog attacks [38].

Notably, media platform preferences and gender were found to be significantly associated with PV. According to the findings of a study by Rogers and Prentice-Dunn [39], a higher quantity of information can increase respondents' PV scores, and Chambers et al. [21] found that low vulnerability may be tied to a lack of knowledge about rabies risks factors and the inability to recognize one's personal risk of rabies. Since YouTube is a popular media platform among students, it can be used to 
disseminate targeted informative videos according to their level of risk. Another study also found that females had a higher degree of PV than males regarding health beliefs and health behaviors [19].

Although this study found that only 3 PMT constructs were associated with RPB, it still cannot be concluded that the nonstatistically significant constructs had no influence. In addition, our model explained only 1 aspect of the total variance of the outcome variable. This indicates that several influential factors were not included and suggests that further research must be conducted.

Another noteworthy finding of this study was its examination of the importance and performance of factors associated with RPB. Our findings suggest that the RE4 ('I think not intervening with animals when they are fighting will help to prevent rabies infection') and S4 indicators in quadrant $D$, which had low performance but high importance, urgently require improvement. As such, the RE4 indicator can be considered a key factor related to RPB. Most students, however, were not aware that avoiding fighting animals is an effective rabies prevention behavior. S4, which was also in quadrant $D$, had a substantial total effect on RPB and a very low performance score. Therefore, S4 and schools in the surrounding area should be the first place where interventions to promote RPB are conducted.

The 3 indicators in quadrant A (RE2: 'I think that informing parents or people about the annual rabies animal vaccination will help to prevent rabies infection,'SE2: 'I am confident that I can reduce my risk of contracting rabies by informing local individuals to vaccinate their pets,' and SE6: 'I am confident that if I do not intervene with fighting dogs, I can reduce my risk of contracting rabies'), which had both high performance and importance, indicate that students already realized that those indicators assisted in rabies prevention. However, because those indicators had very high total effects on the dependent variable, they should still be reinforced in future education programs, particularly RE2 and SE2, in order to encourage students to talk to family and community members about vaccinating their pets against rabies on an annual basis, which they already understood to be effective.

The 2 indicators in quadrant B (PV3: 'If after being bitten you wash the wound with clean water and soap several times for at least 15 minutes, you will have a greater chance of not contracting a rabies infection,' and SE3: 'I am confident that if I immediately inform my teachers/parents after being bitten, scratched, or licked by a mammal, I can reduce my risk of contract- ing rabies'), which had high performance but low importance, indicate that those indicators were not important for RPB. Students' scores for those indicators were still good, however, especially for SE3, which had the highest performance score out of all of the indicators. This indicator ultimately has little influence on RPB. Nevertheless, it is still important to promote education related to these indicators to students in addition to promoting education related to the RE4, RE2, SE2, and SE6 indicators. Boy and YouTube indicators were in quadrant $C$ and had both low performance and importance. Even though those indicators can be considered low-priority for improving RPB, it may still be useful to use YouTube as a tool for the dissemination of rabies education in future prevention and control programs.

This study has some limitations. First, the data for this study were collected using a cross-sectional survey. Therefore, the relationship that was observed between the PMT sub-constructs and other variables needs to be verified in future longitudinal studies. Second, since the participants in this study were fourthgrade students, some PMT constructs, such as perceived rewards and perceived response cost, were not investigated. In future studies of families, these constructs should be added since perceived rewards are known to influence threat appraisal and perceived response cost is known to influence coping appraisal [39].

The results of this study indicate that PMT is a useful framework for predicting behaviors, especially concerning individuals' intentions and motivation related to RPB. Future studies about RPB among school students that are based on PMT should focus on increasing RE, especially for the RE4 indicator and RE2 indicators. In addition, the improvement of SE should also be emphasized so that students feel encouraged to inform family and townspeople of the benefits of annual vaccination against rabies for their pets (SE2) and avoid animals that are fighting (SE6). The results of the present study showed a highly significant relationship between PMT and RPB. If students have more confidence to act, they would be more likely to adopt rabies prevention tactics in high-risk situations [40]. The PMT concepts that were shown to be most influential can be used when designing tools and implementing and evaluating educational interventions to prevent rabies among children.

\section{CONFLICT OF INTEREST}

The authors have no conflicts of interest associated with the material presented in this paper. 


\section{FUNDING}

This project was supported by the Faculty of Public Health at Burapha University.

\section{ACKNOWLEDGEMENTS}

The authors would like to acknowledge the students, families, and teachers for their cooperation. Our great appreciation is also extended to Associate Professor Wathna Soonthorndhai and Lecturer John Daly (USA) for their time, supervision, useful discussion, critical reading, and editing of the manuscript.

\section{AUTHOR CONTRIBUTIONS}

Conceptualization: ML, NM, AW, PA. Data curation: ML. Formal analysis: ML. Funding acquisition: ML, NM, AW, PA. Methodology: ML, NM, AW, PA. Project administration: ML. Visualization: ML. Writing - original draft: ML. Writing - review \& editing: ML, NM, AW, PA.

\section{ORCID}

Mayurin Laorujisawat https://orcid.org/0000-0003-0119-5770

Nipa Maharachpong https://orcid.org/0000-0003-2838-1805

\section{REFERENCES}

1. Centers for Disease Control and Prevention. Global rabies work; 2018 [cited 2019 Mar 21]. Available from: https://www. cdc.gov/ncezid/stories-features/global-stories/rabies-work. html.

2. World Health Organization. Epidemiology and burden of disease [cited 2021 Jan 18]. Available from: https://www.who. int/activities/improving-data-on-rabies/rabies-epidemiology-and-burden.

3. Janchai S. Rabies and situation in Thailand; 2018 [cited 2021 Mar 27]. Available from: https://he02.tci-thaijo.org/index. php/reg45/article/view/123248/93701 (Thai).

4. Bureau of Epidemiology Department of Disease Control. Summary of guidelines for analysis of surveillance systems in 5 groups of diseases in 5 dimensions. 1st ed. Bangkok: TS Interprint; 2016, p. 20-26 (Thai).

5. Rine RC, Dogara GS, Pam MD. Knowledge, attitude and practice of rabies in and around Lafia Metropolis, Nigeria. Cent Afr
J Public Health 2017;3(3):27-33.

6. Deressa A, Ali A, Bayene M, Selassie BN, Yimer E, Hussen K. The status of rabies in Ethiopia: a retrospective record review. Ethiop J Health Dev 2010;24(2):127-132.

7. Lungten L, Rinchen S, Tenzin T, Phimpraphai W, de Garine-Wichatitsky M. Knowledge and perception of rabies among school children in rabies endemic areas of South Bhutan. Trop Med Infect Dis 2021;6(1):28.

8. Sancheti PV, Mangulikar SK. An interventional study to assess knowledge regarding rabies in secondary school students. Int J Community Med Public Health 2016;3(1):180-183.

9. Amparo AC, Mendoza EC, Licuan DA, Valenzuela LM, Madalipay JD, Jayme SI, et al. Impact of integrating rabies education into the curriculum of public elementary schools in llocos Norte, Philippines on rabies knowledge, and animal bite incidence. Front Public Health 2019;7:119.

10. Dzikwi AA, Ibrahim AS, Umoh JU. Knowledge, attitude and practice about rabies among children receiving formal and informal education in Samaru, Zaria, Nigeria. Glob J Health Sci 2012;4(5):132-139.

11. Xiao H, Li S, Chen X, Yu B, Gao M, Yan H, et al. Protection motivation theory in predicting intention to engage in protective behaviors against schistosomiasis among middle school students in rural China. PLoS Negl Trop Dis 2014;8(10):e3246.

12. Rogers RW. A protection motivation theory of fear appeals and attitude change1. J Psychol 1975;91(1):93-114.

13. Clubb AC, Hinkle JC. Protection motivation theory as a theoretical framework for understanding the use of protective measures. Crim Justice Stud (Abingdon) 2015;28(3):336-355.

14. Nutcharporn P. Motivation in the disease prevention by taking the dog to get rabies vaccination among people of Klongplu subdistrict, Nongyai district, Chonburi province [dissertation]. Chonburi: Burapha University; 2016 (Thai).

15. Ezati Rad R, Mohseni S, Kamalzadeh Takhti H, Hassani Azad M, Shahabi N, Aghamolaei T, et al. Application of the protection motivation theory for predicting COVID-19 preventive behaviors in Hormozgan, Iran: a cross-sectional study. BMC Public Health 2021;21(1):466.

16. Roozbahani N, Kaviani AH, Khorsandi M. Path analysis of skin cancer preventive behavior among the rural women based on protection motivation theory. BMC Womens Health 2020; 20(1):121.

17. Sabzmakan L, Ghasemi M, Asghari Jafarabadi M, Kamalikhah T, Chaleshgar Kordasiabi M. Factors associated with tobacco use among Iranian adolescents: an application of protection mo- 
tivation theory. Subst Use Misuse 2018;53(9):1511-1518.

18. Sadeghi R, Mazloomy Mahmoodabad SS, Fallahzadeh H, Rezaeian M, Bidaki R, Khanjani N. Predictive factors for preventing hookah smoking and health promotion among young people based on the protection motivation theory. J Educ Health Promot 2019;8:169.

19. Macdonell K, Chen X, Yan Y, Li F, Gong J, Sun H, et al. A protection motivation theory-based scale for tobacco research among Chinese youth. J Addict Res Ther 2013;4:154.

20. Gong J, Stanton B, Lunn S, Deveaux L, Li X, Marshall S, et al. Effects through 24 months of an HIV/AIDS prevention intervention program based on protection motivation theory among preadolescents in the Bahamas. Pediatrics 2009;123(5):e917e928.

21. Chambers RS, Rosenstock S, Lee A, Goklish N, Larzelere F, Tingey $L$. Exploring the role of sex and sexual experience in predicting American Indian adolescent condom use intention using protection motivation theory. Front Public Health 2018; 6:318.

22. Pham V, Nguyen $H$, Tho le $H$, Minh TT, Lerdboon $P$, Riel $R$, et al. Evaluation of three adolescent sexual health programs in $\mathrm{Ha}$ Noi and Khanh Hoa Province, Vietnam. AIDS Res Treat 2012; 2012:986978.

23. Mwagwabi F, Jiow JH. Compliance with security guidelines in teenagers. Australas J Inf Syst 2021. doi: https://doi.org/10.3127/ ajis.v25i0.2953.

24. World Health Organization. Life skills education for children and adolescents in schools; 1997 [cited 2021 Jan 18]. Available from: https://apps.who.int/iris/handle/10665/63552.

25. Surapong S. Concepts and theories about knowledge attitude and behavior. Bangkok: Chulalongkorn University Press; 1990, p. 218-219 (Thai).

26. Memon MA, Ramayah T, Cheah JH, Ting H, Chuah F, Cham TH. PLS-SEM statistical programs: a review. J Appl Struct Equ Modeling 2021;5(1):i-xiv.

27. SmartPLS GmbH. Importance-performance map analysis (IPMA) [cited 2021 Jan 18]. Available from: https://www.smartpls. com/documentation/algorithms-and-techniques/ipma.

28. Markazi-Moghaddam N, Kazemi A, Alimoradnori M. Using the importance-performance analysis to improve hospital information system attributes based on nurses' perceptions. Inform Med Unlocked 2019;17:100251.

29. Mohd Salleh MI, Abdullah R, Zakaria N. Extending health information system evaluation with an importance-performance map analysis; 2017 [cited 2021 Jan 18]. Available from: https: //www.intechopen.com/chapters/54844.

30. Aeyels D, Seys D, Sinnaeve PR, Claeys MJ, Gevaert S, Schoors D, et al. Managing in-hospital quality improvement: an importance-performance analysis to set priorities for ST-elevation myocardial infarction care. Eur J Cardiovasc Nurs 2018;17:535542.

31. Faul F, Erdfelder E, Lang AG, Buchner A. G*Power 3: a flexible statistical power analysis program for the social, behavioral, and biomedical sciences. Behav Res Methods 2007;39:175191.

32. Bloom BS. Learning for mastery. instruction and curriculum. Regionaleducation laboratory for the carolinas and virginia, topical papers and reprints, number 1; 1968 [cited 2021 Jan 18]. Available from: https://eric.ed.gov/?id=ED053419.

33. Wu HH, Shieh J. Quantifying uncertainty in applying importance-performance analysis. Qual Quant 2010;44(5):997-1003.

34. Kumsri S. Health beliefs affecting rabies disease prevention behavior in the local outbreaks at Hua Sai sub-district Bang Khla district Chachoengsao province. J Depart Med Serv 2018; 43(2):137-140 (Thai).

35. Lanthip $H$, Natthisa B. The perception of information and health behaviors of rabies in Rayong. ODPC6 Chonburi J 2016;7:38-50 (Thai).

36. Thai Rabies Net. Animal rabies surveillance database system [cited 2021 Jan 18]. Available from: http://www.thairabies. net/trn/ (Thai).

37. Arkom M. The relationship between planned behavior and behavior to prevent rabies among people in Paknum-Samutprakarn municipality Bangmuang sub-district of Muang district, Samutprakarn province [dissertation]. Chonburi: Burapha University; 2017 (Thai).

38. Okuhara T, Okada H, Kiuchi T. Predictors of staying at home during the COVID-19 pandemic and social lockdown based on protection motivation theory: a cross-sectional study in Japan. Healthcare (Basel) 2020;8:475.

39. Rogers RW, Prentice-Dunn S. Protection motivation theory. In: Gochman D, editor. Handbook of health behavior research I: personal and social determinants. New York: Plenum Press; 1997, p. 113-132.

40. Sharifirad G, Yarmohammadi P, Sharifabad MA, Rahaei Z. Determination of preventive behaviors for pandemic influenza A/H1N1 based on protection motivation theory among female high school students in Isfahan, Iran. J Educ Health Promot 2014;3:7. 